\title{
Microscopy Study of Xanthorrhoea glauca Leaves and Preliminary Investigation into Biogenic Synthesis of Silver Nanoparticles
}

\author{
Monaliben Shah1, Gordon Thomson², Gérrard Eddy Jai \\ Poinern ${ }^{1}$, Derek Fawcett ${ }^{1}$
}

\begin{abstract}
${ }^{1}$ Murdoch Applied Nanotechnology Research Group. Department of Physics, Energy Studies and Nanotechnology, School of Engineering and Energy, Murdoch University, Murdoch, Western Australia 6150, Australia.

${ }^{2}$ School of Veterinary and Life Sciences, Agricultural Sciences Murdoch University, Murdoch, Western Australia 6150, Australia.
\end{abstract}

\begin{abstract}
The present study examines an eco-friendly room temperature method for synthesising producing Ag nanoparticles using the phytochemicals present in indigenous Xanthorrhoea glauca leaves. The study investigated the transverse and longitudinal sections of the long reed-like leaves via optical microscopy and scanning electron microscopy to determine its structure. Microscopy was also used to determine the sites of nanoparticle synthesis within the leaf structure. While the leaf extract itself was also used to produce $\mathrm{Ag}$ nanoparticles from $\mathrm{AgNO}_{3}$. Particle shapes produced during the 20 minute incubation period consisted of cubes, truncated triangular and hexagonal plates ranging in size from $50 \mathrm{~nm}$ and $200 \mathrm{~nm}$. Both energy dispersive spectroscopy (EDS) and transmission electron microscopy (TEM) were used to confirm Ag nanoparticle formation.
\end{abstract}

Keywords: silver nanoparticles, biogenic synthesis, green chemistry

\section{Introduction}

Nanoparticles exhibit new and unique chemical, physical, optical and electronic properties compared to their macro scale equivalents [1]. These distinctive properties are derived from specific characteristics such as particle size, distribution and shape. Noble metal nanoparticles such as gold $(\mathrm{Au})$, silver $(\mathrm{Ag})$ and platinum $(\mathrm{Pt})$ have been widely used in a variety of fields such as biotechnology, medicine, optoelectronics, pharmaceuticals and materials engineering [2, 3]. Furthermore, nanoparticles have been widely used in commercial products such as detergents, cosmetics, shampoos and even toothpastes [4]. Historically, the disinfecting properties of $\mathrm{Ag}$ have been well known and have been used in past traditional medicinal practices. In recent times Ag nanoparticles have shown themselves to be non-toxic towards humans and effective antimicrobial agents against bacteria, fungi and viruses [5]. The exact antimicrobial mechanism involved is not fully understood, but it is believed the Ag nanoparticles size, shape and surface chemistry interacts and damages both the membrane and nucleic acids of the microbe [6]. Because of the attractive antimicrobial and its anti-inflammatory properties, Ag nanoparticles are currently being used in a variety of commercial pharmaceutical products especially with the advent of antimicrobial resistance to common antibiotics [7, 8]. Advancement of this field not only requires the control of predetermined physiochemical properties, but also on the use of ecofriendly methods to synthesise nanoparticles. Traditional chemical and physical approaches used to produce nanoparticles tend to be complex, use toxic chemicals and have low material conversions. Furthermore, these approaches are generally not ecofriendly and present a wide range of hazards [9]. The use of biological entities and methods employing microorganisms or plant extracts offers a green chemistry based approach that is a viable eco-friendly alternative to traditional chemical and physical approaches [10-12]. Phytochemicals present in plant extracts have the potential to act as both bio-reducing agents and bio-capping agents. The presence of these chemical agents make plant extracts an acceptable eco-friendly method for synthesising $\mathrm{Ag}$

This article is published under the terms of the Creative Commons Attribution License 4.0 Author(s) retain the copyright of this article. Publication rights with Alkhaer Publications. Published at: http://www.ijsciences.com/pub/issue/2016-03/

DOI: 10.18483/ijSci.970; Online ISSN: 2305-3925; Print ISSN: 2410-4477 
nanoparticles. Reviewing the literature reveals an extensive selection of plant extracts have been investigated and employed to synthesise a variety of nanoparticles [13-15]. These studies have revealed that nanoparticle formation is dependent on parameters such as phytochemicals present in the plant species and their concentration, $\mathrm{pH}$, reaction time and temperature. The interplay between these various parameters have been found to directly influence nanoparticle properties such as size, size distribution, surface chemistry and morphology [16].

In the present study, microscopy techniques were used to investigate the indigenous leaf structure of Xanthorrhoea glauca, location of particle synthesis and resulting various nanoparticle properties such as size and shape. The aqueous based technique was found to be straightforward, eco-friendly and produced no toxic waste products. During synthesis silver nitrate was reduced at room temperature to form stable Ag nanoparticles. The nanoparticles were subsequently characterized using UV-visible spectroscopy, transmission electron microscopy (TEM), scanning electron microscopy (SEM) and energy dispersive X-ray spectroscopy (EDS) to determine particle size, morphology and composition.

\section{Materials and Methods}

\subsection{Materials}

Silver nitrate solution $\left[\mathrm{AgNO}_{3},(99.99 \%)\right]$ analytical grade was used as received from Sigma-Aldrich (Castle Hill, NSW, Australia) and all aqueous solutions were prepared from Milli- ${ }^{\circledR}$ water produced from a Barnstead Ultrapure Water System (D11931 - Thermo Scientific Dubuque IA 18.3 M $\Omega$ $\left.\mathrm{cm}^{-1}\right)$.

\subsection{Leaf preparation}

Healthy Xanthorrhoea glauca pencil thin leaves, (at the Murdoch University, South Street campus) were harvested from around the dense crown of leaves protruding outwards from the head of the trunk as seen in Figure 1 (a). The selected leaves were then washed several times in Milli- ${ }^{\circledR}$ water to remove coarse surface impurities. After cleaning, a selection of Xanthorrhoea glauca leaves were prepared for dipping into $\mathrm{AgNO}_{3}$ solutions with the aim of tracking and identifying nanoparticle formation sites within the leaf. The second batch of Xanthorrhoea glauca leaves $(10 \mathrm{~g})$ were finely cut into small strips and placed into a blending bowl containing $100 \mathrm{~mL}$ of Milli-Q ${ }^{\circledR}$ water. The aqueous mixture was homogenized using IKA ${ }^{\circledR}$ T25 Digital Ultra-Turrax ${ }^{\circledR}$ Homogenizer at $5000 \mathrm{rpm}$ for $10 \mathrm{~min}$ at room temperature $\left(24{ }^{\circ} \mathrm{C}\right)$. After homogenization the mixture was filtered (Hirsch funnel) to re-move leaf debris, before two further filtrations using a $0.22 \mu \mathrm{m}$ Millex® (33 mm Dia.) syringe filter unit were carried out. After filtration the leaf extract was placed in clean glass vials ready for nanoparticle synthesis.

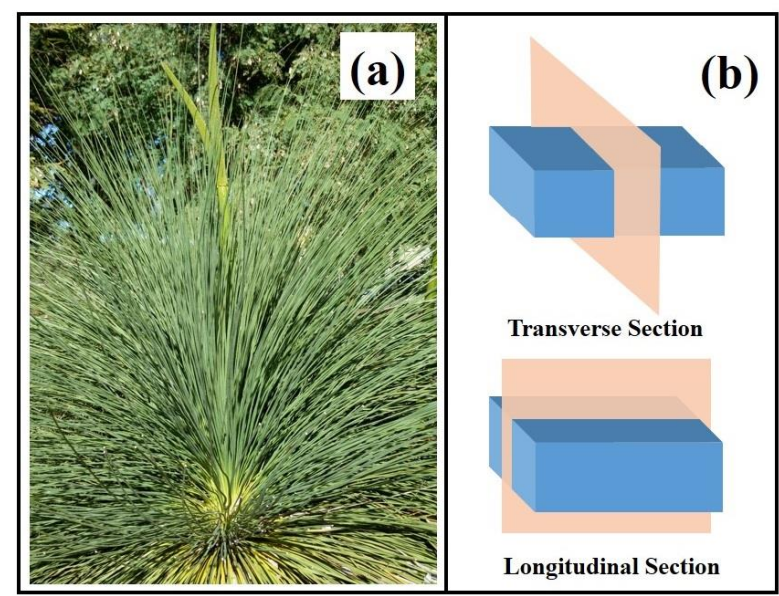

Figure 1 (a) photographic image of Xanthorrhoea glauca Plant (b) Leaf sectioning orientations used in microscopy studies.

\subsection{Nanoparticle Synthesis}

Prior to biosynthesis at room temperature $\left(24^{\circ} \mathrm{C}\right)$, several $1 \mathrm{~mL}$ solutions of $0.1 \mathrm{M} \mathrm{AgNO}_{3}$ were prepared using Milli- $\mathrm{Q}^{\circledR}$ water. The individual $\mathrm{AgNO}_{3}$ solutions were added to three leaf extracts consisting of $1 \mathrm{~mL}$ for $\mathrm{s} 1,2 \mathrm{~mL}$ for $\mathrm{s} 2$ and $3 \mathrm{~mL}$ for $\mathrm{s} 3$. The various mixtures were agitated for 1 minute before being permitted to stand, during which time the colour change of each respective mixture was monitored. In the dipping tests, leafs were vertically placed into $1 \mathrm{~mL}$ solutions of $0.1 \mathrm{M} \mathrm{AgNO}_{3}$ and progress of $\mathrm{AgNO}_{3}$ permeation was monitored using microscopy.

\subsection{Optical Microscopy, Scanning Electron} Microscopy and Energy Dispersive Spectroscopy

Prior to optical microscopy, Johansen's Safranin O and Fast Green stains were used to stain the respective sectioned Xanthorrhoea glauca leaf material. While Formalin-Acetic-Alcohol (FAA) was used as a fixing agent to preserve the leaf sections. Leaf material was sectioned in two planes (transverse and longitudinal) as shown in Figure 1 (b). The leaf sections were studied using a Olympus BX51 compound microscope (Olympus Optical Co. Ltd., Tokyo, Japan) and were photographed with the integrated DP 70 camera attachment. Leaf sections were also studied using characterisation techniques such as SEM, TEM and EDS to determine particle elemental analysis, particle size and morphology. TEM particle analysis consisted of placing a single drop of reaction medium onto a carbon-coated copper TEM grid. The sample was then allowed to dry over a 24 hours period before undergoing examination. 
The bright field study was carried out using a Phillips CM-100 electron microscope operating at $80 \mathrm{kV}$ (Phillips Corporation Eindhoven, The Netherlands). Leaf sections were examined using a NeoScope ${ }^{\mathrm{TM}}$ (JCM-6000) SEM fitted with a secondary energy dispersive spectrometer. Samples were mounted on SEM stubs using carbon tape and then coated with a $2 \mathrm{~nm}$ layer of gold using a Cressington 208HR sputter coater to improve electrical conductivity.
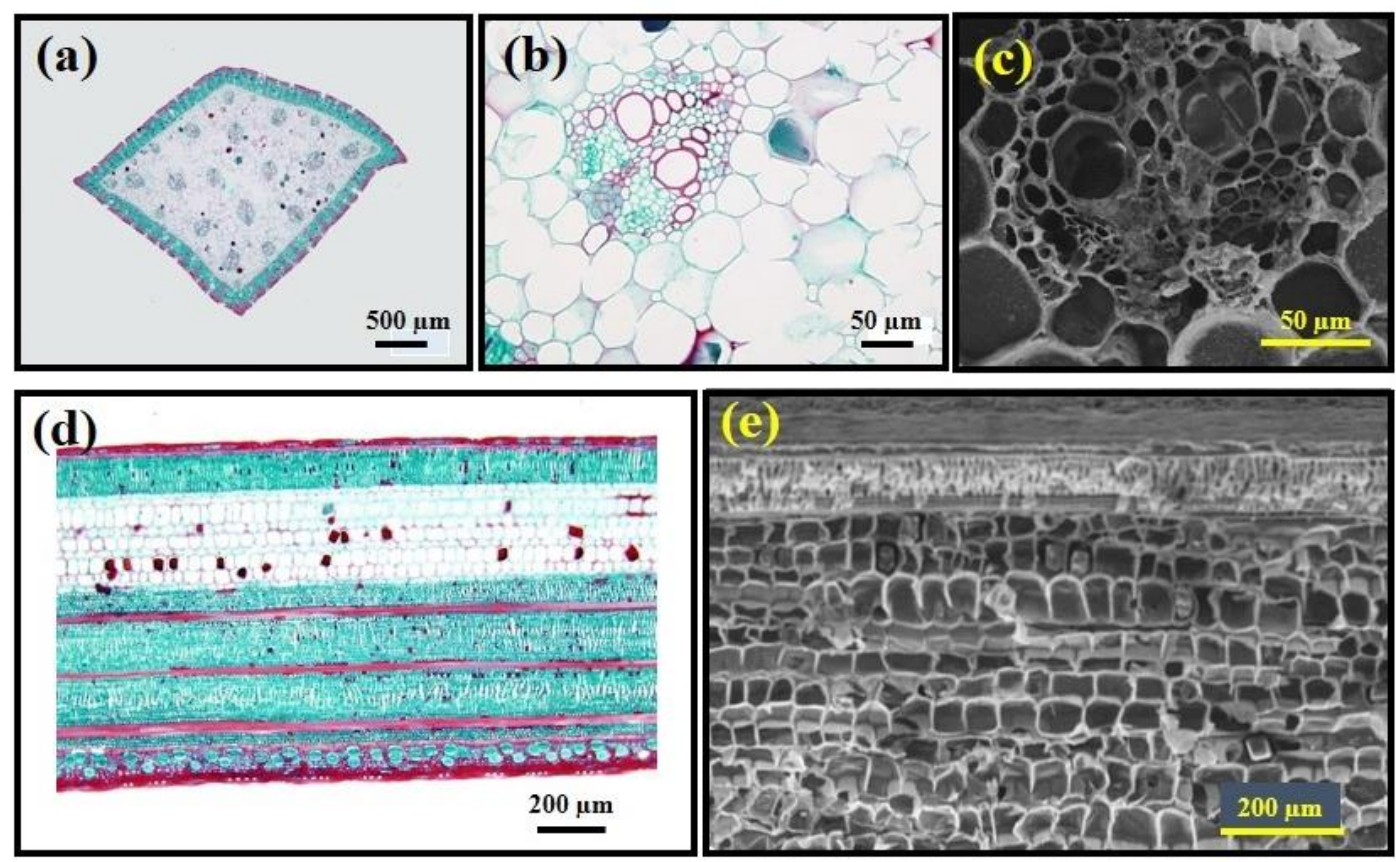

Figure 2 (a) typical optical image of transverse section of leaf clearly showing vascular system; (b) and (c) optical and SEM images of a representative vascular bundle (phloem \& xylem), and (d) and e) optical and SEM longitudinal sections of surface and inner leaf structure.

\section{Results and Discussions}

Figure 1 presents optical and SEM microscopy images of sectioned leaf material in transverse and longitudinal planes. Figure 2 (a) presents a representative optical image of a transverse leaf section showing its internal structure and overall square shape. The image reveals a waxy cuticle and epidermis layers covering a relative thin layer of palisade mesophyll cells that contain a large interior composed of spongy mesophyll cells surrounding an array of vascular bundles. Figures 2 (b) and 2 (c) present optical and SEM images of a typical vascular bundle that is used to transport xylem (water and minerals) and phloem (food energy) throughout the leaf. Figures 2 (d) and 2 (e) present optical and SEM images of a representative longitudinal section showing the internal structure of a leaf. In the optical images Safranin stain appears as a brilliant red in chromosomes, nuclei and cutinized cell walls. While the Fast green stains colours the cytoplasm and cellulosic cell walls. During the dipping procedure $\mathrm{AgNO}_{3}$ could be visually seen moving up the leaf (yellow arrow) via a very noticeable colour change as seen in Figure 3 (a). Initially the $\mathrm{AgNO}_{3}$ solution was clear but within 20 minutes it had become black, indicating bio-reduction had taken place. Optical microscopy revealed that $\mathrm{AgNO}_{3}$ enters the leaf via stomata and vascular bundles (red arrows) as seen in Figure 3 (b) and (c). However, much larger quantities of $\mathrm{AgNO}_{3}$ end up in the region of the cells than the vascular bundles (red arrows) as seen in Figures (b) and (d). Enlarged images presented in Figures 4 (a) and (b) also indicates the biosynthesis of $\mathrm{Ag}$ nanoparticles (red arrows) via the reduction of $\mathrm{AgNO}_{3}$ occurs predominantly at the cell walls. 

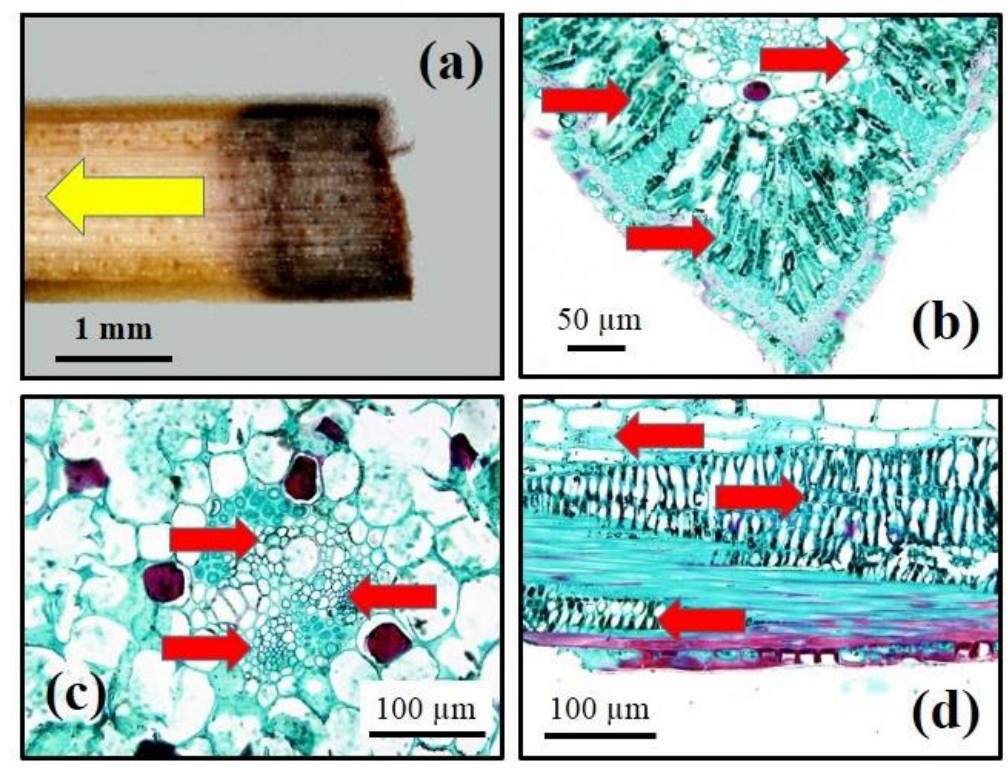

Figure 3 (a) leaf after dipping in $\mathrm{AgNO}_{3}$; (b) and (c) transverse sectional images showing principle regions of $\mathrm{AgNO}_{3}$ reduction to form Ag nanoparticles, and (d) longitudinal section showing Ag NPs along the cells.

TEM analysis of the samples after 20 minutes revealed the biosynthesis process had produced particles that had an anisotropic character. Particle shapes found in representative samples include bars (blue circle), truncated triangular (yellow circle) and hexagonal plates (red circle) as seen in Figure 4 (c). Also present in smaller numbers were cubes and spherical shapes. The nanoparticles ranged in size between $50 \mathrm{~nm}$ and $200 \mathrm{~nm}$, with mean size around $100 \mathrm{~nm}$. Nanoparticles biosynthesised using the pure leaf extract were also found to produce particles with similar shapes and size range. EDS elemental compositional analysis conducted after biosynthesis indicated the presence of metallic $\mathrm{Ag}$ in the samples. The very strong signal for metallic $\mathrm{Ag}$, as seen in Figure 4 (d) confirmed the results of the TEM study that clearly indicated the presence of $\mathrm{Ag}$ nanoparticles.
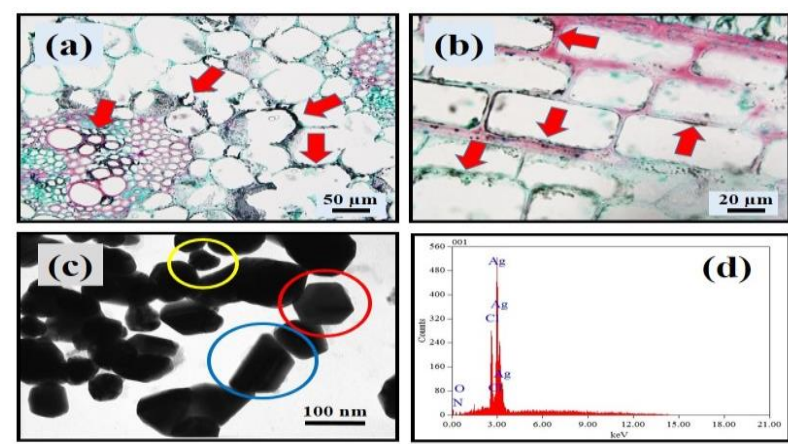

Figure 4 Enlarged views of leaf showing actual sites of Ag nanoparticle biosynthesis (a) transverse section and (b) longitudinal section; (c) representative TEM image of Ag nanoparticles produced, and (d) EDS analysis confirming the presence of metallic Ag nanoparticles.

This preliminary study has revealed that indigenous Xanthorrhoea glauca leaves contains the necessary phytochemicals needed to act as both reducing agent and stabilising agent during the synthesis of $\mathrm{Ag}$ nanoparticles. Initial results indicate that phytochemicals present in the cell membranes were effective in reducing $\mathrm{AgNO}_{3}$ solutions to form $\mathrm{Ag}$ nanoparticles. The size and shape of nanoparticles formed in the leaf were found to be similar to those formed when an aqueous solution of $\mathrm{AgNO}_{3}$ was mixed with a leaf extract solution. The resultant reaction mixture after 20 minutes produced nanoparticles of similar shape and size range as those produced in the leaf as seen in Figure 5 (a). Clearly seen in Figure 5 (a) are the hexagonal plates and a truncated triangular plate highlighted by a yellow circle. In addition, the elemental compositional analysis after a 20 minute incubation period revealed a the strong $\mathrm{Ag}$ peak indicating $\mathrm{Ag}$ nanoparticle formation had taken place as seen in Figure 5 (b). The results of the preliminary study indicate leaf material of Xanthorrhoea glauca has the ability to reduce and produce stable Ag nanoparticles in situ directly from $\mathrm{AgNO}_{3}$ solutions, (even without having to make an extract). However, further studies are needed to identify the exact phytochemicals involved in nanoparticle reduction and those involved in stabilizing the nanoparticles. Also, further studies are needed to investigate potential antimicrobial 

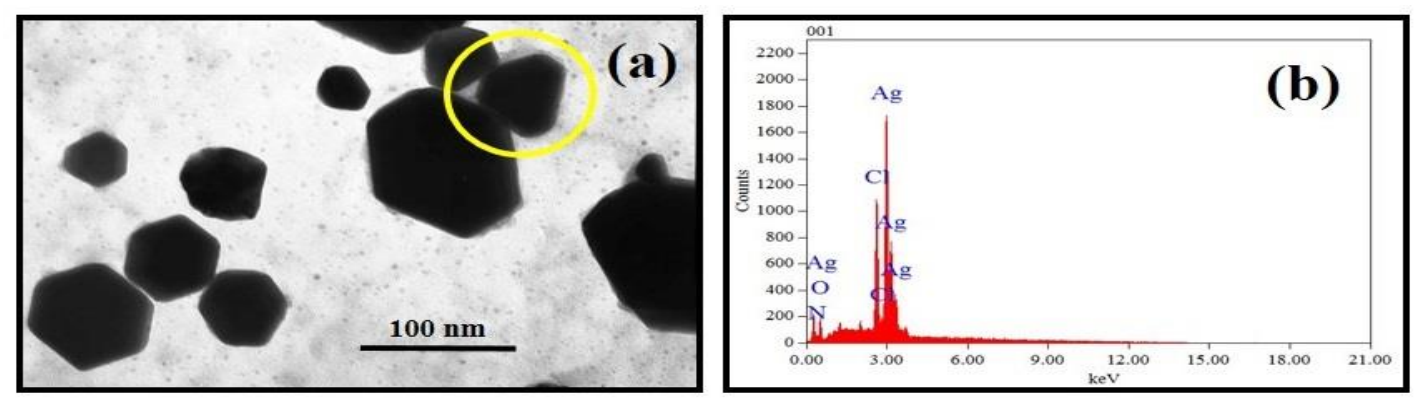

Figure 5 (a) $\mathrm{Ag}$ nanoparticles formed via $\mathrm{AgNO}_{3} /$ leaf extract reaction solutions and (b) EDS elemental analysis confirming metallic $\mathrm{Ag}$ nanoparticle formation via reaction mixture

\section{Conclusion}

The present study has examined an eco-friendly room temperature method for producing Ag nanoparticles via the phytochemicals present in Xanthorrhoea glauca leaves. The phytochemicals present in the cell membranes of the leaf were able to reduce $\mathrm{Ag}$ nanoparticles from $\mathrm{AgNO}_{3}$ solutions, then coat and stabilise the nanoparticles. The formed nanoparticles were anisotropic in nature and were similar to those synthesised in $\mathrm{AgNO}_{3}$ /leaf extract mixtures after a 20 minute incubation time. Nanoparticle shapes included cubes, truncated triangular and hexagonal plates ranging in size from $50 \mathrm{~nm}$ and $200 \mathrm{~nm}$. However, further research is needed to identify individual phytochemicals involved in $\mathrm{Ag}$ nanoparticle formation, physiochemical properties of the nanoparticles and potential applications such as antimicrobial pharmaceuticals.

\section{Acknowledgements}

This work was partly supported by Horticulture Innovation Australia Project Al14003 and Dr Derek Fawcett would like to thank Horticulture Innovation Australia for their research fellowship.

\section{Disclosure}

The authors claim no conflict of interest in this work.

\section{References}

1) Zaniewski, A. M., Schriver, M., Lee, J. G., Crommie, M. F., Zettl, A. Electronic and optical of metal nanoparticles filled graphene sandwiches. J. Appl. Phys. Lett. 2013; 102: 023108, $1-5$.

2) Salata, O. V. Applications of nanoparticles in biology and medicine. Journal of Nanobiotechnology. 2004; 2 (3): 1-6.

3) Alshehri, A. H., Jakubowska, M., Młożniak, A., Horaczek, M., Rudka, D., Free, C., Carey, J. D. Enhanced Electrical Conductivity of Silver Nanoparticles for High Frequency Electronic Applications. ACS Appl. Mater. Interfaces, 2012; 4 (12): 7007-7010.

4) Jeong, S. H., Yeo, S. Y., Yi, S. C. The effect of filler particle size on the antibacterial properties of compounded polymer/silver fibres. J. Mat. Sci. 2005; 40: 5407-5411.

5) Choi, O., Deng, K. K., Kim, N. J., et al. The inhibitory effects of silver nanoparticles, silver ions, and silver chloride colloids on microbial growth, Water Res. 2008; 42: $3066-$ 3074.

6) Wong, K. K. Y., Liu, X. L. Silver nanoparticles: the real "silver bullet" in clinical medicine? Med. Chem. Comm. 2010; 1: 125-131.

7) Li, W. R., Xie, X. B., Shi, Q. S., Duan, S. S., Ouyang, Y. S. Chen, Y. B. Antibacterial effect of silver nanoparticles on Staphylococcus aureus. Biometals 2011; 24 (1): 135-141.

8) Cox, S. G., Cullingworth, L., Rode, H. Treatment of paediatricburns with a nanocrystalline silver dressing compared with standard wound care in a burns unit: a cost analysis. South African Medical Journal. 2011; 101 (10): 728-731.

9) Luyts, K., Napierska, D., Nemery, B., Hoet, P. H. M. How physico-chemical characteristics of nanoparticles cause their toxicity: complex and unresolved interrelations. Environ. Sci. Processes Impact. 2013; 15: 23-38.

10) Lengke, M., Southam, G. Bioaccumulation of gold by sulphate-reducing bacteria cultured in the presence of gold (I)-thiosulfate complex. Acta. 2006; 70 (14): 3646-3661.

11) Shanmugavadivu, M., Kuppusamy, S., Ranjithkumar, R. Synthesis of pomegranate peel extract mediated silver nanoparticles and its antibacterial activity. Am. J. Adv. Drug. Deliv. 2014; 2 (2): 174-182.

12) Kumar, P., Singh, P., Kumari, K., et al. A green approach for the synthesis of gold nanotriangles using aqueous leaf extract of Callistemon viminalis. Mater. Lett. 2011; 65: 595-597.

13) Jacob, J., Mukherjee, T., Kapoor, S. A simple approach for facile synthesis of $\mathrm{Ag}$, anisotropic $\mathrm{Au}$ and bimetallic $(\mathrm{Ag} / \mathrm{Au})$ nanoparticles using cruciferous vegetable extracts. Materials Science and Engineering: C. 2012; 32: 1827-1834.

14) Poinern, G. E. J., Le, X., Chapman, P., Fawcett, D. Green biosynthesis of gold nanoparticles using the leaf extracts from an indigenous Australian plant Eucalyptus macrocarpa. Gold Bulletin 2013; 46: 165-173.

15) Sheny, D. S., Mathew, T., Philip, D. Phytosynthesis of Au, $\mathrm{Ag}$ and $\mathrm{Au}-\mathrm{Ag}$ bimetallic nanoparticles using aqueous extract and dried leaf of Anacardium occidentale. Spectrochim. Acta Part A. 2011; 79: 254-262.

16) Mittal, A. K., Chisti, Y., Banerjee, U. C. Synthesis of metallic nanoparticles using plants. Biotechnology Advances. 2013; 31: 346-356. 